\title{
Antagonism of CXCR7 attenuates chronic hypoxia-induced pulmonary hypertension
}

Ecaterina Sartina', Cleide Suguihara', Shalini Ramchandran', Patrick Nwajei', Myra Rodriguez'1, Eneida Torres', Dorothy Hehre', Carlos Devia', Matthew J. Walters' ${ }^{2}$, Mark E.T. Penfold ${ }^{2}$ and Karen C. Young ${ }^{1}$

INTRODUCTION: Chemokines may directly participate in the pathogenesis of neonatal chronic hypoxia-induced pulmonary hypertension (PH). Although stromal-derived factor-1 (SDF-1) has been shown to be involved in $\mathrm{PH}$, the role of its most recently discovered receptor, chemokine receptor type 7 (CXCR7), remains unclear. We sought to determine whether antagonism of the CXCR7 receptor would decrease pulmonary vascular remodeling in newborn mice exposed to chronic hypoxia by decreasing pulmonary vascular cell proliferation.

METHODS: Neonatal mice were exposed to hypoxia (fractional inspired oxygen concentration $=0.12$ ) or room air (RA) for $2 \mathrm{wk}$. After 1 wk of exposure, mice received daily injections of placebo or a CXCR7 antagonist (CCX771) from postnatal day 7 (P7) to P14. Right ventricular systolic pressure (RVSP), the ratio of the weight of the right ventricle to left ventricle + septum (RV/LV $+\mathrm{S})$, and pulmonary vascular cell proliferation and remodeling were determined at P14.

RESULTS: As compared with mice exposed to RA, hypoxia placebo mice had a significant increase in the lung protein expression of CXCR7. Although hypoxic placebo-treated mice had a significant increase in RVSP, RV/LV+S, and pulmonary vascular cell proliferation and remodeling, the administration of CCX771 markedly decreased these changes.

DISCUSSION: These results indicate that antagonism of CXCR7 may be a potent strategy to decrease PH and vascular remodeling.

$\mathbf{P}$ ulmonary hypertension (PH) affects $>1$ in 1,000 neonates/y and remains a significant cause of morbidity and mortality in preterm and term neonates (1). This disease occurs secondary to various insults during the newborn period, such as perinatal hypoxia, sepsis, and meconium aspiration, and as a complication of chronic lung disease of prematurity (1). Remodeling of the pulmonary arterioles is a hallmark of PH. This is characterized by smooth muscle cell hypertrophy or hyperplasia, extension of muscularization into the walls of normally nonmuscular peripheral pulmonary arteries, and adventitial thickening. The increased tone in the newly muscularized arteries induces increased pulmonary vascular resistance, resulting in elevation of pulmonary arterial pressure and right ventricular hypertrophy. The combined effect of these changes leads to the development of $\mathrm{PH}$ and ultimately right-cardiac failure.

Inflammation has been suggested to play a significant role in the pathogenesis of $\mathrm{PH}(2,3)$. Chemokines are peptides that orchestrate the migration of cells involved in inflammatory responses (4). Based on the relative position of the $\mathrm{N}$-terminal cysteine residues, chemokines are classified into four main families: $\mathrm{CX}_{3} \mathrm{C}, \mathrm{CXC}, \mathrm{C}$, and CC chemokines (4). The CXC chemokine family is a pleiotropic family of cytokines that is involved in many essential biological processes, including cell differentiation, proliferation, and apoptosis, as well as angiogenesis; these cytokines are known to play a pivotal role in the regulation of leukocyte trafficking into sites of tissue inflammation (5-7).

CXC chemokine receptor type 7 (CXCR7) is a recently deorphanized G protein-coupled receptor. The ligands for CXCR7 are stromal-derived factor-1 (SDF-1) or CXC chemokine ligand 12 (CXCL12) and interferon-inducible T-cell a-chemoattractant (CXCL11; refs. 8,9). This is important, as SDF-1 has been shown to significantly participate in the pathogenesis of neonatal chronic hypoxia-induced PH by its regulation of progenitor cell migration as well as pulmonary vascular cell proliferation and survival (10). Although CXCR4, the only other receptor for SDF-1, has also been shown to participate in the development of PH $(10,11)$, the role of CXCR7 in chronic hypoxia-induced pulmonary vascular remodeling is not entirely known. CXCR7 has, however, been previously demonstrated to be highly expressed on activated pulmonary arterial endothelial cells $(12,13)$ as well as vascular smooth muscle cells (14). Moreover, this receptor has been shown to play an important role in cell proliferation, survival, and adhesion $(9,15-17)$ - processes that are important for the development of $\mathrm{PH}$.

We, therefore, tested the hypothesis that CXCR7 would be increased in the lungs of neonatal mice with $\mathrm{PH}$ and, furthermore, that antagonism of CXCR7 would decrease neonatal chronic hypoxia-induced $\mathrm{PH}$ and pulmonary vascular cell proliferation and remodeling. 


\section{RESULTS}

CXCR7 Expression Is Increased in the Lungs of Neonatal Mice With Chronic Hypoxia-Induced PH

We first examined the expression of CXCR7 in lysates made from the lungs of newborn mice. Following chronic hypoxiainduced $\mathrm{PH}$, there was a 1.5 -fold increase in the expression of CXCR7 in the lungs of placebo (PL)-treated mice and this was markedly decreased following the administration of CCX771 (Figure 1a). Immunohistochemical staining showed little CXCR7 in normal mouse lungs but revealed extensive CXCR7 mainly localized to the endothelium and adventitia of the remodeled hypoxic murine pulmonary arteriole (Figure 1b; nonimmune IgG control shown at right). This finding is in agreement with prior data showing that CXCR7 RNA is selectively upregulated in hypoxic lungs in vivo (18). Furthermore, in keeping with previously published data (10), exposure of neonatal mice to chronic hypoxia markedly increased the expression of the CXCR7 ligands, SDF-1, and CXCL11, as shown by enzyme-linked immunosorbent assay and western blot analyses, respectively, of lung homogenates in Figure 1c,d.
Of note, although the administration of CCX771 had no effect on lung SDF-1 concentration (Figure 1c), this antagonist decreased the expression of CXCL11 (Figure 1d).

\section{Antagonism of CXCR7 Attenuates Chronic Hypoxia-Induced PH} and Pulmonary Vascular Cell Remodeling

We next asked whether antagonism of CXCR7 could be a strategy to decrease $\mathrm{PH}$. PH was induced in neonatal mice by exposure to chronic hypoxia from postnatal day 1 (P1) to P14, and randomly assigned animals received daily injections of CCX771 or PL from P7 to P14. As compared with mice exposed to room air (RA), chronically hypoxic mice had a significant increase in right ventricular systolic pressure (RVSP; $10 \pm 1$ vs. $20 \pm 3 \mathrm{~mm}$ Hg; RA-PL vs. Hypoxia-PL, $P<0.001)$ as well as the ratio of the right ventricle to the left ventricle + septum $(\mathrm{RV} / \mathrm{LV}+\mathrm{S} ; 0.3 \pm 0.1$ vs. $0.5 \pm 0.1$, RA-PL vs. Hypoxia-PL, $P<0.001$; Figure 2a,b). In contrast, in the CCX771-treated animals, there was no hypoxiainduced increase in RVSP (20 \pm 3 vs. $11 \pm 3$ mm Hg; Hypoxia-PL vs. Hypoxia CCX771, $P<0.001)$ or RV/LV + S (0.5 \pm 0.1 vs. 0.3 \pm 0.2 ; Hypoxia-PL vs. Hypoxia CCX771, $P<0.01$; Figure 2a,b),
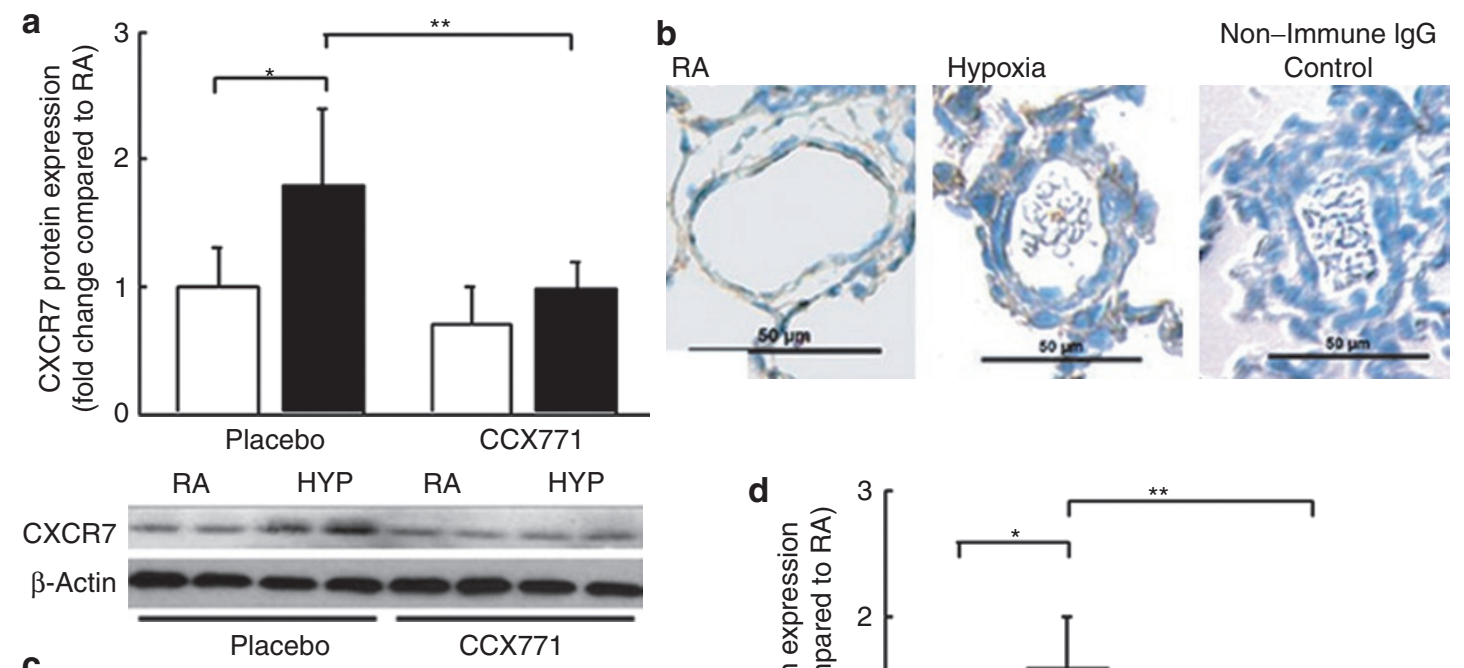

d
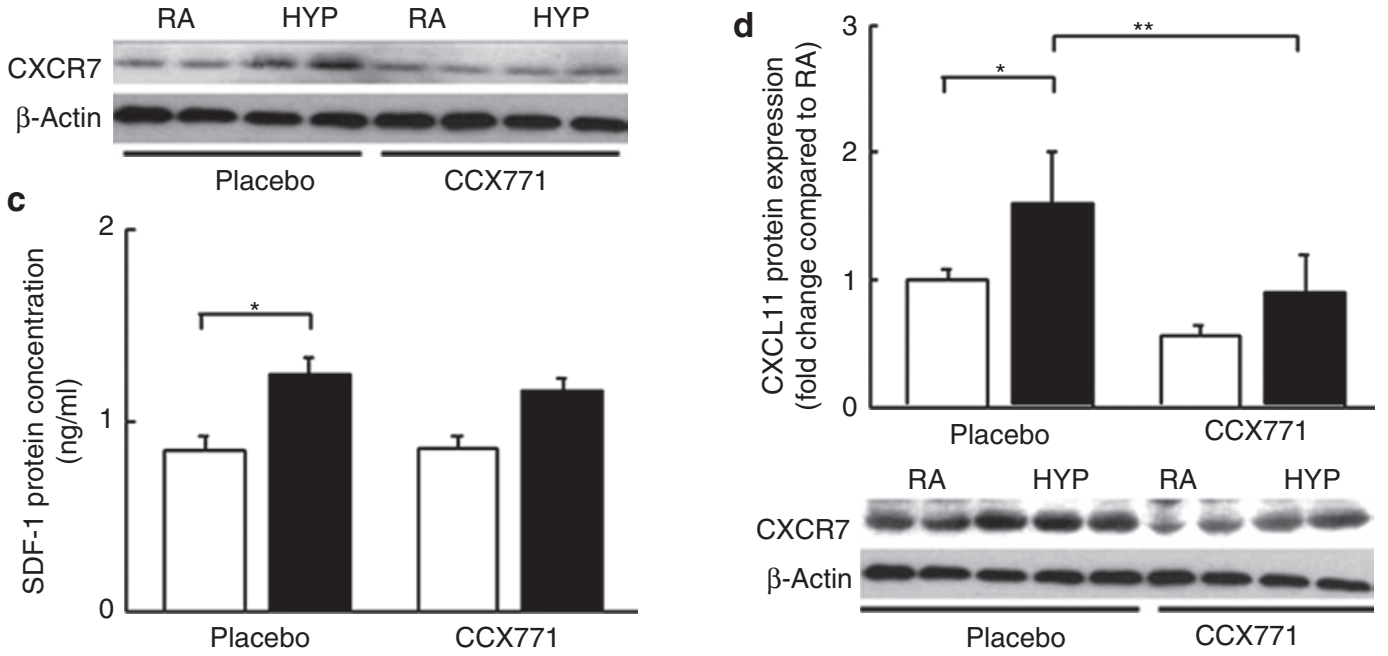

Figure 1. CXCR7 expression is increased in the lungs of neonatal mice with chronic hypoxia-induced pulmonary hypertension (PH). (a) Increased CXCR7 protein expression in the lungs of newborn mice with chronic hypoxia (HYP)-induced PH ( $P<0.05 ; n=4-5 /$ group). Administration of CCX771 decreased

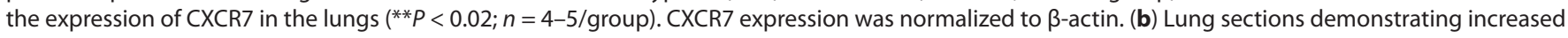
CXCR7 staining in the pulmonary vascular endothelium and adventitia of hypoxic mice (brown staining). Nonimmune lgG control is shown in the right panel. Original magnification: $\times 400$. (c) SDF- 1 protein expression is increased in the lungs of mice with chronic hypoxia-induced $\mathrm{PH}\left({ }^{*} P<0.005\right.$; $n=4-5$ /group). Administration of CCX771 does not affect SDF-1 concentration. (d) CXCL11 protein expression is increased in the lungs of mice with chronic hypoxia-induced $\mathrm{PH}\left({ }^{*} P<0.05 ; n=4-5 /\right.$ group). Administration of CCX771 decreased the expression of CXCL11 in the lungs $\left({ }^{* *} P<0.002\right.$; $n=4-5$ /group). CXCL11 expression was normalized to $\beta$-actin. CCX771, CXCR7 antagonist; CXCL11, CXC chemokine ligand 11; CXCR7, chemokine receptor type 7; RA, room air; SDF-1, stromal-derived factor-1. 

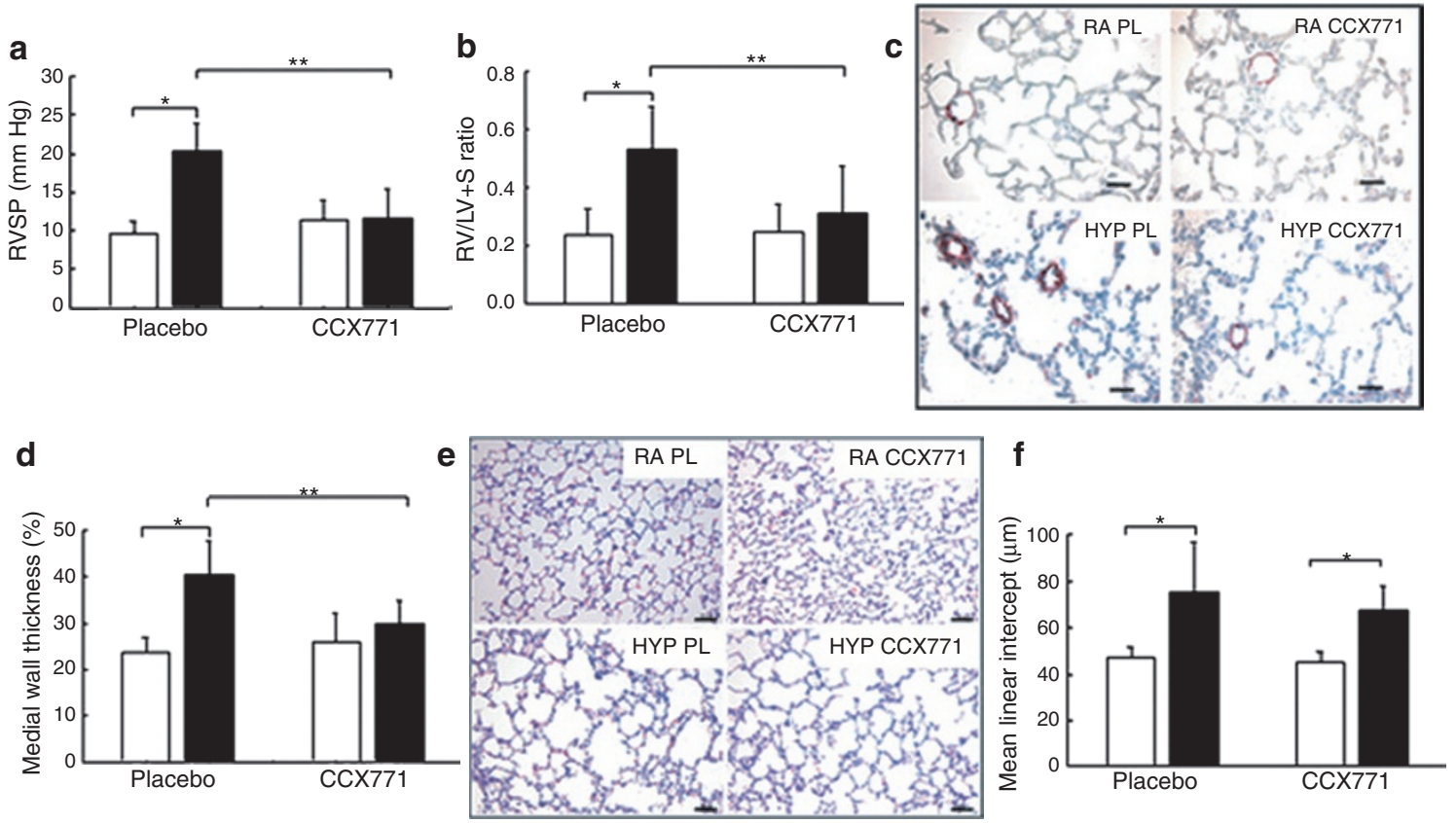

Figure 2. Antagonism of CXCR7 attenuates PH and vascular remodeling. (a) CCX771 decreased RVSP in chronically hypoxic mice ( $* P<0.001, \mathrm{RA}-\mathrm{PL}$ vs. Hypoxia-PL and ${ }^{* *} P<0.001, C C X 771$ vs. PL; $n=16-22 /$ group). White bars represent RA and black bars represent hypoxia. (b) CCX771 decreased RV/LV $+\mathrm{S}$ in chronically hypoxic mice $\left({ }^{*} P<0.001\right.$, RA-PL vs. Hypoxia-PL and ${ }^{* *} P<0.007, C C X 771$ vs. $P$ L; $n=5-9 /$ group). (c) Lung sections demonstrating decreased a-smooth muscle actin staining in hypoxic CCX771-treated mice. Original magnification: $\times 400$. Bar $=25 \mu \mathrm{m}$. (d) CCX771 decreased the medial wall thickness of pulmonary arterioles in chronically hypoxic mice $\left({ }^{*} P<0.001\right.$; RA-PL vs. Hypoxia-PL and ${ }^{* *} P<0.017, \mathrm{CCX771}$ vs. PL; $n=5-8 /$ group). (e) Hematoxylin and eosin-stained lung sections demonstrating worsened alveolarization in chronically hypoxic mice. CCX771 does not affect lung alveolarization. Original magnification: $\times 200$. Bar $=50 \mu \mathrm{m}$. (f) Mean linear intercept was increased in the hypoxia-placebo-treated mice $\left({ }^{*} P<0.001 ; \mathrm{RA}-\mathrm{PL}\right.$ vs. Hypoxia-PL and RA-CXC771 vs. Hypoxia-CCX771; $n=5-8$ /group). CCX771, CXCR7 antagonist; CXCR7, chemokine receptor type 7; HYP, hypoxia; PH, chronic hypoxiainduced pulmonary hypertension; $\mathrm{PL}$, placebo; RA, room air; RV/LV + S, ratio of right ventricle to left ventricle + septum; RVSP, right ventricular systolic pressure.

suggesting that inhibition of CXCR7 could abrogate the effects of hypoxia on these measures.

In accordance with these findings, there was also a significant increase in the pulmonary vascular medial wall thickness of chronically hypoxic mice $(0.2 \pm 0.03$ vs. $0.4 \pm 0.07$; RA-PL vs. Hypoxia-PL, $P<0.001$; Figure $2 \mathrm{c}$,d) and concomitant increased expression of $\alpha$-smooth muscle actin. In contrast, administration of CCX771 to chronically hypoxic mice significantly inhibited the pulmonary arteriolar medial wall thickening ( $0.4 \pm 0.03$ vs. $0.3 \pm 0.05$; Hypoxia-PL vs. Hypoxia CCX771, $P<0.001$; Figures $2 \mathrm{c}$,d). These findings suggest that antagonism of CXCR7 can partially abrogate chronic hypoxiainduced $\mathrm{PH}$ and pulmonary vascular remodeling.

Because chronic hypoxic exposure also decreases alveolarization in the developing lung (19), we next asked whether antagonism of CXCR7 would improve lung alveolarization. Although hypoxic mice had increased mean linear intercept, indicating worsening alveolarization $(P<0.001$; RA-PL vs. Hypoxia-PL; $n=5-8$ /group), these metrics were unaffected following administration of the CXCR7 antagonist (Figure 2e,f).

\section{Antagonism of CXCR7 Attenuates Pulmonary Vascular Proliferation \\ We next questioned whether CXCR7 mediates chronic hypoxia-induced pulmonary vascular cell proliferation.}

Sections of lung from newborn mice were stained for the proliferative marker Ki67, and a proliferative index was calculated as described in the Methods section. Chronically hypoxic mice had a significant increase in the pulmonary vascular proliferative index $(0.09 \pm 0.03$ vs. $0.59 \pm 0.04$; RA-PL vs. Hypoxia-PL, $P<0.001$; Figure 3a,b). In contrast, no such increase was observed in the hypoxic CCX771-treated mice $(0.59 \pm 0.04$ vs. $0.24 \pm 0.1$; Hypoxia-PL vs. Hypoxia CCX771, $P<0.01)$. These findings suggest that the antagonism of CXCR7 may modulate $\mathrm{PH}$ and pulmonary vascular remodeling by decreasing the pulmonary vascular cell proliferation that leads to the disease.

Antagonism of CXCR7 Attenuates Pulmonary Vascular Adhesion CXCR7 has been shown to modulate the adhesion of leukocytes to the endothelium (20). Inflammatory cell migration and adhesion are integral processes in chronic hypoxia-induced pulmonary vascular remodeling (21). We, therefore, investigated whether CXCR7 would modulate the expression of the known adhesion molecules intracellular adhesion molecule-1 (ICAM-1) and vascular cell adhesion molecule-1 (VCAM-1). Tissue homogenates were prepared from mouse lungs and assessed by western blot. Chronically hypoxic mice had a significant increase in the expression of the adhesion molecules ICAM-1 (2.5-fold; RA-PL vs. Hypoxia-PL, $P<0.03$; Figure 4a) and VCAM-1 (1.5-fold; RA-PL vs. Hypoxia-PL, $P<0.007$; 
a
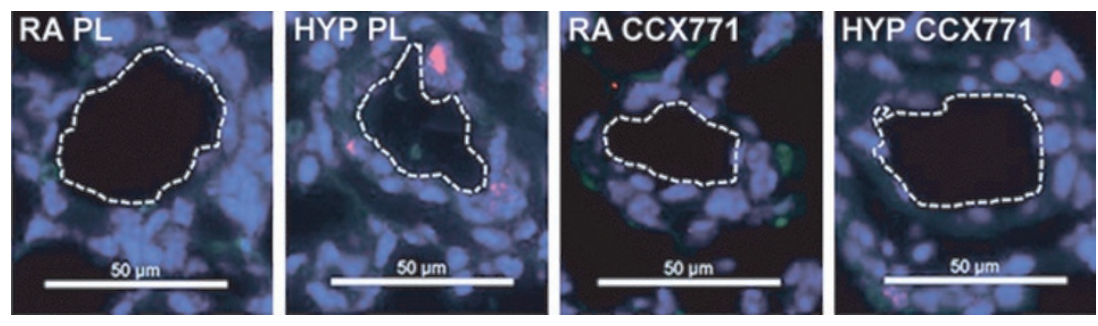

b

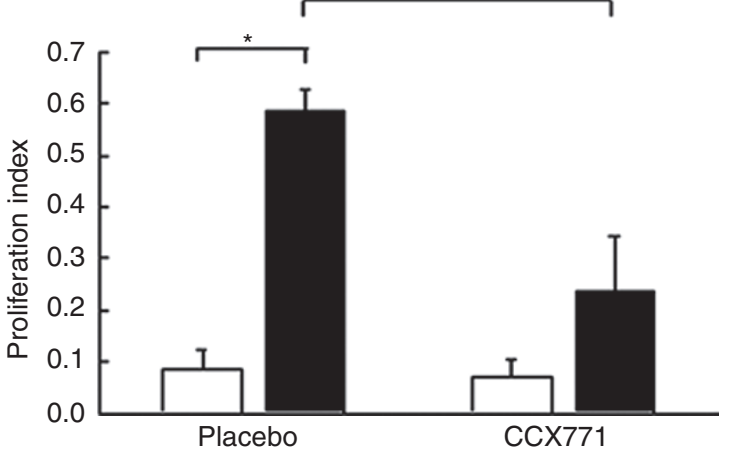

Figure 3. Antagonism of CXCR7 decreases pulmonary vascular proliferation. (a) Representative immunofluorescence images of pulmonary arterioles following staining with anti-Ki67 antibody (red) and 4',6-diamidino-2-phenylindole (blue). Original magnification: $\times 400$. (b) Administration of CCX771 markedly decreased chronic hypoxia-induced pulmonary vascular cell proliferation $\left({ }^{*} P<0.001, \mathrm{RA}-\mathrm{PL}\right.$ vs. Hypoxia-PL and ${ }^{* *} P<0.01, \mathrm{CCX} 771$ vs. $\mathrm{PL} ; n=4 /$ group). CCX771, CXCR7 antagonist; CXCR7, chemokine receptor type 7; HYP, hypoxia; PL, placebo; RA, room air.
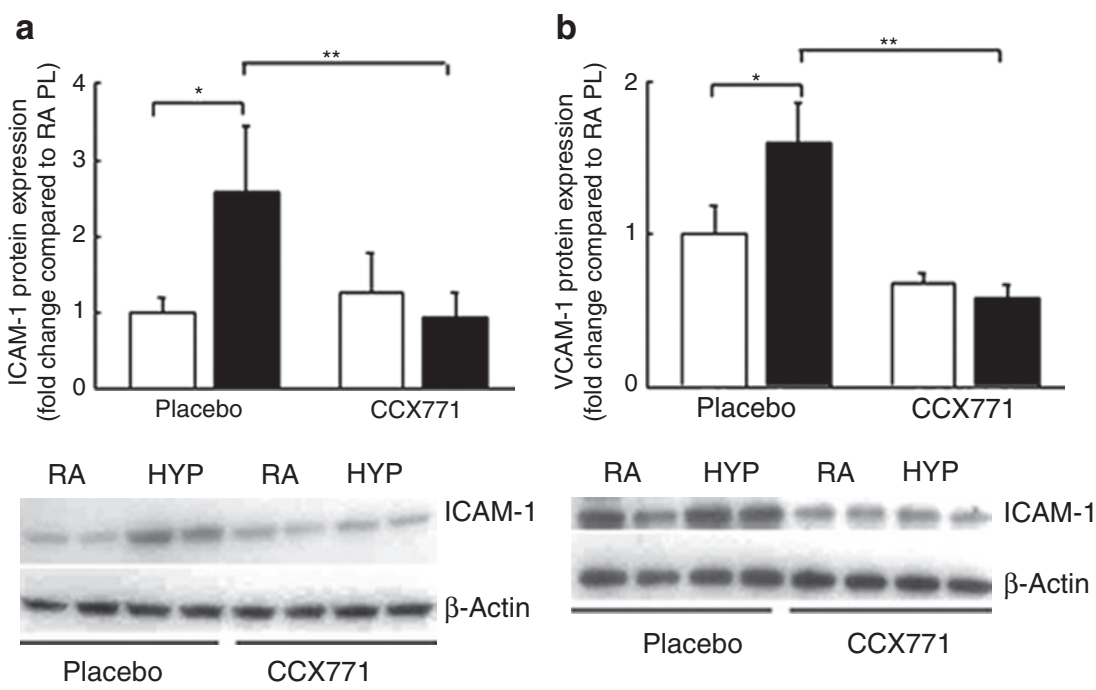

Figure 4. Antagonism of CXCR7 decreases pulmonary vascular adhesion. (a) CCX771 decreases ICAM-1 expression $\left({ }^{*} P<0.03\right.$, RA-PL vs. Hypoxia-PL and ${ }^{* *} P<0.003, C C X 771$ vs. PL; $n=4-5 /$ group). (b) CCX771 decreases VCAM-1 expression ( ${ }^{*} P<0.007$, RA-PL vs. Hypoxia-PL and ${ }^{*} P<0.001, C C X 771$ vs. PL; $n=4-5$ /group). CCX771, CXCR7 antagonist; CXCR7, chemokine receptor type 7; HYP, hypoxia; ICAM-1, intracellular adhesion molecule-1; PL, placebo; RA, room air; VCAM-1, vascular cell adhesion molecule-1.

Figure 4b). In contrast, expression of these proteins was not increased in the hypoxic CCX771-treated mice, suggesting that antagonism of CXCR7 may decrease chronic hypoxia-induced pulmonary vascular remodeling by decreasing cell adhesion.

\section{DISCUSSION}

$\mathrm{PH}$ and right heart failure is a significant cause of mortality and morbidity in preterm and term neonates (1). This study sought to elucidate whether CXCR7, a newly deorphanized receptor for SDF-1, participates in $\mathrm{PH}$ by increasing pulmonary vascular cell proliferation. We first demonstrated that CXCR7 protein is markedly increased in the pulmonary arterioles of chronically hypoxic neonatal mice with $\mathrm{PH}$, in agreement with a previous study demonstrating an upregulation of CXCR7 RNA following hypoxia in vivo (18). Moreover, antagonism of CXCR7 significantly decreased the amount of hypoxia-induced $\mathrm{PH}$, right ventricular hypertrophy, and pulmonary vascular cell proliferation and 


\section{Articles | Sartina et al.}

remodeling. These findings provide new evidence for a role of CXCR7 in the pathogenesis of $\mathrm{PH}$ and, of note, suggest that CXCR7 antagonism may be used as a novel strategy to treat chronic hypoxia-induced $\mathrm{PH}$ in neonates.

Hypoxic exposure of the pulmonary vascular bed has been shown to induce a prominent inflammatory response within the vessel wall (11). Indeed, within $48 \mathrm{~h}$ of hypoxic exposure, mice were shown to have a prominent infiltration of neutrophils and macrophages in the lung and bronchoalveolar fluid (22). Furthermore, overexpression of tumor necrosis factor- $\alpha$ in mice led to pathological and physiological findings consistent with severe PH (23), whereas administration of the antiinflammatory cytokine interleukin-10 prevented the development of PH in rats and improved their survival (24).

Among molecules known to mediate the inflammatory response are chemokines (4), of which CXCR7 is a member of the CXC subgroup of chemokine receptors (8). Although expression patterns for this receptor remain controversial $(8,9,13,25,26)$, there is general agreement that it can be expressed on the membranes of endothelial cells and smooth muscle cells in various tissues $(9,16,20,27)$. The ligands for CXCR7 are CXCL11 and SDF-1 (ref. 9). The signaling pathways that are activated following the binding of CXCR7 to its ligands are still controversial. Indeed, some studies suggest that CXCR7 is a nonsignaling, decoy receptor that scavenges and degrades chemokine ligands $(28,29)$. However, although there is evidence to suggest that CXCR7 does not signal through classical G-protein-mediated pathways $(14,28)$, many studies have demonstrated an important role of this receptor in physiological and pathological processes $(9,25,30-32)$. CXCR7-deficient mice were shown to die within the first week after birth due to cardiovascular malformations (25). Moreover, within several organ systems, CXCR7 has been shown to play a distinctive role in the adhesion, proliferation, and migration of endothelial, smooth muscle, and progenitor cells $(20,31)$. This study not only demonstrates an increase in the expression of CXCR7 and its ligands in the lungs of neonatal mice with $\mathrm{PH}$ but also provides new insight into a functional role of CXCR7 in regulating $\mathrm{PH}$, possibly through modulation of pulmonary vascular cell proliferation, adhesion, and remodeling.

This finding is in keeping with our previous study showing that antagonism of SDF-1 or its other receptor, CXCR4, could attenuate $\mathrm{PH}$ in neonatal mice (10). This is also consistent with the observations of Gambaryan et al. (13), who demonstrated that the CXCR4 antagonist AMD3100 inhibited hypoxia-induced pulmonary vascular remodeling in an adult model and showed synergistic effects when combined with a CXCR7 antagonist. This latter finding is particularly relevant, given the data suggesting that the CXCR7/CXCR4 heterodimer potentiates SDF-1-mediated downstream $\beta$-arrestindependent cell signaling pathways (33). It should be noted, however, that although Gambaryan et al. did not observe any significant effects on pulmonary vascular remodeling following the administration of the CXCR7 antagonist alone, differences in the age and strain of mice (the C57/BL6 strain used in Gambaryan's study is genetically deficient in the gene for the CXCR7 ligand CXCL11; ref. 34), and hence possible differences in the mechanisms and pathology of disease, preclude direct comparisons.

In our model of PH, antagonism of CXCR4 or CXCR7 comparably attenuated neonatal chronic hypoxia-induced $\mathrm{PH}$ (10). It should, however, be noted that although CXCR4 is expressed on inflammatory as well as bone marrow-derived progenitor cells (35), both significant contributors to the pathogenesis of PH (36), CXCR7 has been shown to modulate the transendothelial migration of inflammatory cells (20). It is, therefore, plausible that CXCR4 and CXCR7 play overlapping as well as distinct roles in the pathogenesis of $\mathrm{PH}$, and thus the relative impact of their antagonists in $\mathrm{PH}$ may be dependent on the disease model as well as the stage of the disease.

This study also demonstrates that the antagonism of CXCR7 significantly decreases chronic hypoxia-induced pulmonary vascular cell proliferation. These findings are in keeping with other studies showing that chemokines may potentiate vascular remodeling by modulating pulmonary vascular cell proliferation (10,37-39). Moreover, CXCR7 has been specifically shown to increase the proliferation of lung microvascular endothelial cells and several tumor lines $(12,15,17)$. Wang et al. demonstrated that overexpression of CXCR7 increased the basal proliferation rates of prostatic cancer cell lines (17), whereas knock-down of CXCR7 decreased the proliferation of mammary carcinoma cells (15). Although the signaling pathways through which CXCR7 may modulate cell proliferation are yet to be elucidated, it has been suggested that CXCR7 might signal through extracellular-signal-regulated kinases, Akt, and protein kinase C pathways (30).

Finally, we observed a positive modulatory effect of CXCR7 on the expression of the adhesion molecules ICAM-1 and VCAM-1. We demonstrated that antagonism of CXCR7 markedly suppressed the upregulation of these adhesion molecules observed in chronically hypoxic neonatal mice. The homing and adhesion of inflammatory cells to the pulmonary vasculature is known to be an important process in pulmonary vascular remodeling $(11,21)$. Elevated levels of the adhesion molecules (VCAM-1 and ICAM-1) have been demonstrated in several animal models of PH (11). Burke et al. demonstrated increased expression levels of the adhesion molecules ICAM-1 and VCAM-1 in the pulmonary arteries of chronically hypoxic rats (11). Furthermore, elevated levels of these adhesion molecules have been demonstrated in children with congenital heart disease and PH (40), whereas amelioration of chronic hypoxiainduced $\mathrm{PH}$ was associated with decreased ICAM-1 expression (41). Although few published reports have demonstrated that CXCR7 directly modulates the expression of these adhesion proteins (20), the involvement of CXCR7 in cell adhesion was shown in several recent studies. Burns et al. demonstrated that CXCR7 transfectant cell lines adhered to activated human umbilical vein endothelial cells markedly more than wild-type cell lines (9), and antagonism of CXCR7 was shown to inhibit the passage of lymphocytes through endothelial monolayers (42). Moreover, Mazzinghi et al. showed that antagonism of 
CXCR7 decreased the adhesion of renal multipotent progenitor cells to endothelial cells (31). It is possible that increased adhesion and/or extravasation of leukocytes mediated by CXCR7 via activated endothelium at sites of inflammation contribute to the pathology of diseases including $\mathrm{PH}$.

This study has a few limitations. Neonatal chronic hypoxia-induced pulmonary vascular remodeling is known to be associated with increased proliferation of pulmonary vascular smooth muscle cells and fibroblasts, with less significant proliferation of endothelial cells. This study showed that CXCR7 has a positive mitogenic effect on pulmonary vascular cells; further investigations will need to be performed to identify the specific pulmonary vascular cell population that is modulated by this receptor. Furthermore, although CCX771 is a specific inhibitor of CXCR7, in this study, we also demonstrated that CCX771 not only decreased the protein expression of CXCR7 but also the expression of its ligand, CXCL11. It is, therefore, possible that some of the effects of CCX771 may have been due to secondary effects on the expression of CXCL11, a known chemoattractant.

Nonetheless, taken together, the findings in this study suggest that CXCR7 may participate in neonatal chronic hypoxiainduced $\mathrm{PH}$ by regulating cell proliferation as well as adhesion. Although the molecular mechanisms by which CXCR7 regulates these responses are still unclear, and further studies will be necessary to define them, this study suggests that antagonism of CXCR7 may be a useful strategy to attenuate PH and pulmonary vascular remodeling.

\section{METHODS}

\section{Animals}

Pregnant female mice (FVB/NJ; $n=16)$ were obtained from Jackson Laboratories (Bar Harbor, ME). The animals were treated according to the National Institutes of Health guidelines for the use and care of laboratory animals and the study protocol was approved by the University of Miami Animal Care and Use Committee.

\section{Study Design}

Newborn pups were randomly assigned to be exposed to normobaric normoxia (RA) or hypoxia (fractional inspired oxygen concentration $=0.12$ ) for 2 weeks. Oxygen concentration was maintained by controlling the inflow rates of nitrogen and oxygen. The mice were monitored with oxygen analyzers (model OM25RME; Maxtec, Salt Lake City, UT). After 1 week of RA or hypoxic exposure, the mice received daily subcutaneous injections of a vehicle (PL) or a specific CXCR7 antagonist, CCX771 $(30 \mathrm{mg} / \mathrm{kg} / \mathrm{dose}$; donated by ChemoCentryx, Mountain View, CA) from P7 to P14. The compound is a potent and specific modulator of CXCR7 that inhibits the binding of SDF-1 and CXCL11 to CXCR7. The dose and interval for the administration of the CCX771 were based on pharmacokinetic studies performed by the manufacturer and pilot studies demonstrating an increase in CXCR7 expression within $7 \mathrm{~d}$ of hypoxic exposure. Litter size was adjusted to seven pups to control for the effect of the litter size on nutrition and growth. Mothers were rotated every $24 \mathrm{~h}$. The degree of $\mathrm{PH}$ and vascular remodeling was evaluated at P14.

\section{Assessment of $\mathrm{PH}$}

The degree of $\mathrm{PH}$ in neonatal mice was determined as previously described (10). RVSP was measured as a surrogate of pulmonary artery pressure and right ventricular hypertrophy was assessed by $\mathrm{RV} / \mathrm{LV}+\mathrm{S}$.

\section{Assessment of Pulmonary Vascular Remodeling}

Pulmonary vascular morphometry was performed as previously described (10). Briefly, a 23-gauge catheter was introduced through the right ventricular wall and advanced into the pulmonary artery. The catheter was connected to a reservoir containing phosphatebuffered saline and potassium cyanide solution. The latter solution ensured that the effects of CCX771 or PL on the vasculature were not confounded by hypoxia-induced vasoconstriction. After completion of the vascular perfusion, a vascular fixative containing $4 \%$ paraformaldehyde was infused. To determine the degree of pulmonary artery muscularization, 5 - $\mu \mathrm{m}$ paraffin-embedded sections were stained with polyclonal rabbit antihuman von Willebrand Factor (1:200; Dako, Carpinteria, CA) and monoclonal mouse anti-a-smooth muscle actin (1:500; Sigma-Aldrich, St Louis, MO). Medial wall thickness of partially and fully muscular arteries $(20-50 \mu \mathrm{m})$ was determined by using the formula: $2 \mathrm{MT} \times 100 / \mathrm{ED}$ (where MT is the distance between internal and external elastic laminas and ED is the external diameter). Approximately 20 randomly chosen arteries were evaluated per slide and all morphometric analyses were performed by a blinded observer.

\section{Assessment of Pulmonary Vascular Cell Proliferation}

The number of proliferating pulmonary vascular arterial cells was determined by staining lung sections with Ki67, a marker of cell proliferation (1:100; Abcam, Cambridge, MA). The proliferation index was calculated by the following formula: No. of Ki67 ${ }^{+}$cells/(No. of $\mathrm{Ki}^{+} 7^{+}+$No. of Ki67 ${ }^{-}$cells per pulmonary arteriole). A total of 20 randomly selected arteries $(20-50-\mu \mathrm{m}$ diameter) were evaluated by a blinded observer.

\section{Western Blot}

The lungs of neonatal FVB/NJ mice exposed to hypoxia or normoxia for $2 \mathrm{wk}$ were dissected as previously described (10). Western blot analysis of whole-lung lysates was performed as previously described (10). The primary antibodies utilized were mouse CXCR7 monoclonal antibody 11G8 (ChemoCentryx); rat ICAM-1 monoclonal antibody (Abcam), and rabbit VCAM-1 polyclonal antibody (Santa Cruz Biotechnology, Santa Cruz, CA).

\section{SDF-1 Enzyme-Linked Immunosorbent Assay}

Lung SDF-1a concentrations from newborn mice lung homogenates were determined using a Quantikine enzyme-linked immunosorbent assay kit (R\&D Systems, Minneapolis, MN) per the manufacturer specifications.

\section{Immunohistochemistry}

Immunostaining was performed as described previously (10). The primary antibodies utilized were rabbit polyclonal Ki67 antibody (Abcam) and mouse monoclonal CXCR7 antibody 11G8 (donated by ChemoCentryx).

\section{Statistics}

All results are reported as mean \pm SD. Data were analyzed by two-way ANOVA followed by a post hoc analysis (Holm-Sidak). Values of $P<0.05$ were considered statistically significant. Statistical analysis was performed using SigmaStat software (SyStat Software, San Jose, CA).

\section{STATEMENT OF FINANCIAL SUPPORT}

This study was supported by Project Newborn; an Ikaria, Advancing Newborn Medicine Grant to E.S.; a National Institutes of Health K08 Award; a Florida Biomedical Research Award; and a Batchelor Research Foundation Award to K.C.Y.

Disclosure: M.J.W. and M.E.T.P are employed by ChemoCentryx, Inc., which has an active program to develop therapeutics that target CXCR7. This declaration is made in the interests of full disclosure and in no way suggests or implies any conflict of interest on the part of the authors. 


\section{REFERENCES}

1. Walsh-Sukys MC, Tyson JE, Wright LL, et al. Persistent pulmonary hypertension of the newborn in the era before nitric oxide: practice variation and outcomes. Pediatrics 2000;105(1 Pt 1):14-20.

2. Tuder RM, Voelkel NF. Pulmonary hypertension and inflammation. J Lab Clin Med 1998;132:16-24.

3. Dorfmüller P, Perros F, Balabanian K, Humbert M. Inflammation in pulmonary arterial hypertension. Eur Respir J 2003;22:358-63.

4. Rollins BJ. Chemokines. Blood 1997;90:909-28.

5. Balabanian K, Foussat A, Dorfmüller P, et al. CX(3)C chemokine fractalkine in pulmonary arterial hypertension. Am J Respir Crit Care Med 2002;165:1419-25.

6. Kucia M, Jankowski K, Reca R, et al. CXCR4-SDF-1 signalling, locomotion, chemotaxis and adhesion. J Mol Histol 2004;35:233-45.

7. Plotkin J, Prockop SE, Lepique A, Petrie HT. Critical role for CXCR4 signaling in progenitor localization and $\mathrm{T}$ cell differentiation in the postnatal thymus. J Immunol 2003;171:4521-7.

8. Balabanian K, Lagane B, Infantino S, et al. The chemokine SDF-1/CXCL12 binds to and signals through the orphan receptor RDC1 in T lymphocytes. J Biol Chem 2005;280:35760-6.

9. Burns JM, Summers BC, Wang Y, et al. A novel chemokine receptor for SDF-1 and I-TAC involved in cell survival, cell adhesion, and tumor development. J Exp Med 2006;203:2201-13.

10. Young KC, Torres E, Hatzistergos KE, Hehre D, Suguihara C, Hare JM. Inhibition of the SDF-1/CXCR4 axis attenuates neonatal hypoxia-induced pulmonary hypertension. Circ Res 2009;104:1293-301.

11. Burke DL, Frid MG, Kunrath CL, et al. Sustained hypoxia promotes the development of a pulmonary artery-specific chronic inflammatory microenvironment. Am J Physiol Lung Cell Mol Physiol 2009;297: L238-50.

12. Costello CM, McCullagh B, Howell K, et al. A role for the CXCL12 receptor, CXCR7, in the pathogenesis of human pulmonary vascular disease. Eur Respir J 2011; e-pub ahead of print 16 November 2011.

13. Gambaryan N, Perros F, Montani D, et al. Targeting of c-kit+ haematopoietic progenitor cells prevents hypoxic pulmonary hypertension. Eur Respir J 2011;37:1392-9.

14. Rajagopal S, Kim J, Ahn S, et al. Beta-Arrestin- but not G protein-mediated signaling by the "decoy" receptor CXCR7. Proc Natl Acad Sci USA 2010;107:628-32.

15. Meijer J, Ogink J, Roos E. Effect of the chemokine receptor CXCR7 on proliferation of carcinoma cells in vitro and in vivo. $\mathrm{Br} \mathrm{J}$ Cancer 2008;99:1493-501.

16. Miao Z, Luker KE, Summers BC, et al. CXCR7 (RDC1) promotes breast and lung tumor growth in vivo and is expressed on tumor-associated vasculature. Proc Natl Acad Sci USA 2007;104:15735-40.

17. Wang J, Shiozawa Y, Wang J, et al. The role of CXCR7/RDC1 as a chemokine receptor for CXCL12/SDF-1 in prostate cancer. J Biol Chem 2008;283:4283-94.

18. Costello CM, Howell K, Cahill E, et al. Lung-selective gene responses to alveolar hypoxia: potential role for the bone morphogenetic antagonist gremlin in pulmonary hypertension. Am J Physiol Lung Cell Mol Physiol 2008;295:L272-84.

19. Vicencio AG, Eickelberg O, Stankewich MC, Kashgarian M, Haddad GG. Regulation of TGF-beta ligand and receptor expression in neonatal rat lungs exposed to chronic hypoxia. J Appl Physiol 2002;93:1123-30.

20. Cruz-Orengo L, Holman DW, Dorsey D, et al. CXCR7 influences leukocyte entry into the CNS parenchyma by controlling abluminal CXCL12 abundance during autoimmunity. J Exp Med 2011;208:327-39.

21. Hassoun PM, Mouthon L, Barberà JA, et al. Inflammation, growth factors, and pulmonary vascular remodeling. J Am Coll Cardiol 2009;54(1 Suppl):S10-9.
22. Minamino T, Christou H, Hsieh CM, et al. Targeted expression of heme oxygenase-1 prevents the pulmonary inflammatory and vascular responses to hypoxia. Proc Natl Acad Sci USA 2001;98:8798-803.

23. Fujita M, Shannon JM, Irvin CG, et al. Overexpression of tumor necrosis factor-alpha produces an increase in lung volumes and pulmonary hypertension. Am J Physiol Lung Cell Mol Physiol 2001;280:L39-49.

24. Ito $\mathrm{T}, \mathrm{Okada} \mathrm{T}$, Miyashita $\mathrm{H}$, et al. Interleukin-10 expression mediated by an adeno-associated virus vector prevents monocrotaline-induced pulmonary arterial hypertension in rats. Circ Res 2007;101:734-41.

25. Gerrits H, van Ingen Schenau DS, Bakker NE, et al. Early postnatal lethality and cardiovascular defects in CXCR7-deficient mice. Genesis 2008;46:235-45.

26. Berahovich RD, Zabel BA, Penfold ME, et al. CXCR7 protein is not expressed on human or mouse leukocytes. J Immunol 2010;185:5130-9.

27. Neusser MA, Kraus AK, Regele H, et al. The chemokine receptor CXCR7 is expressed on lymphatic endothelial cells during renal allograft rejection. Kidney Int 2010;77:801-8.

28. Boldajipour B, Mahabaleshwar H, Kardash E, et al. Control of chemokineguided cell migration by ligand sequestration. Cell 2008;132:463-73.

29. Naumann U, Cameroni E, Pruenster M, et al. CXCR7 functions as a scavenger for CXCL12 and CXCL11. PLoS ONE 2010;5:e9175.

30. Odemis V, Boosmann K, Heinen A, Küry P, Engele J. CXCR7 is an active component of SDF-1 signalling in astrocytes and Schwann cells. J Cell Sci 2010;123(Pt 7):1081-8.

31. Mazzinghi B, Ronconi E, Lazzeri E, et al. Essential but differential role for CXCR4 and CXCR7 in the therapeutic homing of human renal progenitor cells. J Exp Med 2008;205:479-90.

32. Sánchez-Martín L, Estecha A, Samaniego R, Sánchez-Ramón S, Vega MÁ, Sánchez-Mateos P. The chemokine CXCL12 regulates monocytemacrophage differentiation and RUNX3 expression. Blood 2011;117:88-97.

33. Décaillot FM, Kazmi MA, Lin Y, Ray-Saha S, Sakmar TP, Sachdev P. CXCR7/CXCR4 heterodimer constitutively recruits beta-arrestin to enhance cell migration. J Biol Chem 2011;286:32188-97.

34. Sierro F, Biben C, Martínez-Muñoz L, et al. Disrupted cardiac development but normal hematopoiesis in mice deficient in the second CXCL12/SDF-1 receptor, CXCR7. Proc Natl Acad Sci USA 2007;104:14759-64.

35. Möhle R, Bautz F, Rafii S, Moore MA, Brugger W, Kanz L. The chemokine receptor CXCR-4 is expressed on $\mathrm{CD} 34^{+}$hematopoietic progenitors and leukemic cells and mediates transendothelial migration induced by stromal cell-derived factor-1. Blood 1998;91:4523-30.

36. Stenmark KR, Fagan KA, Frid MG. Hypoxia-induced pulmonary vascular remodeling: cellular and molecular mechanisms. Circ Res 2006;99:675-91.

37. Yue TL, Wang X, Sung CP, et al. Interleukin-8. A mitogen and chemoattractant for vascular smooth muscle cells. Circ Res 1994;75:1-7.

38. Sanchez O, Marcos E, Perros F, et al. Role of endothelium-derived CC chemokine ligand 2 in idiopathic pulmonary arterial hypertension. Am J Respir Crit Care Med 2007;176:1041-7.

39. Perros F, Dorfmüller P, Souza R, et al. Fractalkine-induced smooth muscle cell proliferation in pulmonary hypertension. Eur Respir J 2007;29:937-43.

40. Sungprem K, Khongphatthanayothin A, Kiettisanpipop P, Chotivitayatarakorn P, Poovorawan Y, Lertsapcharoen P. Serum level of soluble intercellular adhesion molecule-1 correlates with pulmonary arterial pressure in children with congenital heart disease. Pediatr Cardiol 2009;30:472-6.

41. Satoh K, Fukumoto Y, Nakano M, et al. Statin ameliorates hypoxia-induced pulmonary hypertension associated with down-regulated stromal cell-derived factor-1. Cardiovasc Res 2009;81:226-34.

42. Zabel BA, Wang Y, Lewén S, et al. Elucidation of CXCR7-mediated signaling events and inhibition of CXCR4-mediated tumor cell transendothelial migration by CXCR7 ligands. J Immunol 2009;183:3204-11. 